\title{
DETERMINANTS OF HEALTHY AGEING AND ITS RELATION TO 10-YEAR CARDIOVASCULAR DISEASE INCIDENCE: THE ATTICA STUDY
}

\author{
Natasa Kollia', Demosthenes B. Panagiotakos ${ }^{1}$, Christina Chrysohoou², Ekavi Georgousopoulou', Dimitrios \\ Tousoulis $^{2}$, Christodoulos Stefanadis ${ }^{2}$, Charalabos Papageorgiou ${ }^{3}$, Christos Pitsavos ${ }^{2}$ \\ ${ }^{1}$ Department of Nutrition and Dietetics, School of Health Science and Education, Harokopio University, Athens, Greece \\ ${ }^{2}$ First Cardiology Clinic, School of Medicine, University of Athens, Athens, Greece \\ ${ }^{3}$ Department of Psychiatry, School of Medicine, University of Athens, Athens, Greece
}

\begin{abstract}
SUMMARY
Objectives: The aim of the study was to understand determinants of ageing in relation to future cardiovascular disease (CVD) events at population level, and to explore determinants of healthy ageing and its relation to 10-year CVD incidence among apparently healthy individuals.

Methods: In the context of the ATTICA study 453 apparently healthy men ( $45 \pm 13$ years) and 400 women ( $44 \pm 18$ years) with complete psychological evaluation were studied and followed during 2002-2012. Healthy Ageing Index (HAI) (range 0-7) was calculated based on socioeconomical, bio-clinical, psychological, and lifestyle characteristics (i.e. dietary habits and physical activity) of the participants during the baseline examination. CVD incidence during the follow-up period was defined according to ICD-10 criteria.

Results: Healthy Ageing Index was inversely associated with higher 10-year CVD risk (OR per 1/7 (95\% Cl): $0.47(0.28,0.80)$. Age and sexadjusted determinants of healthy ageing were abnormal waist to hip ratio $(p<0.001)$, increased coffee consumption $(p=0.04)$, reduced basic metabolic rhythm $(p<0.001)$, increased triglycerides $(p=0.003)$, and $C$-reactive protein levels $(p=0.02)$, as aggravating factors, while moderate alcohol consumption $(p=0.002)$ was identified as a positive influential parameter.

Conclusions: Understanding healthy ageing, as a dominant factor of CVD development, provides a new direction for better prevention efforts focused on healthy ageing at both population and individual level.
\end{abstract}

Key words: ageing, cardiovascular disease, risk factors, psychological

Address for correspondence: Demosthenes B. Panagiotakos, 46 Paleon Polemiston St., Glyfada, Attica, 166 74, Greece. E-mail: d.b.panagiotakos@ usa.net

https://doi.org/10.21101/cejph.a5165

\section{INTRODUCTION}

During the last decades life expectancy has substantially improved throughout the world, and centenarians are becoming a fast-growing group of the population. According to the World Health Organization (WHO), the older adult population will continue to grow, posing a public health challenge to societies and undermining the importance of a life course approach to "healthy ageing". The increase in the proportion of older people is the result of unprecedented economic, social, medical, and technological changes that has made it possible for people to live a long and, probably, active life. In 2006, the European Commission identified demographic ageing as one of the most challenging policy issues of the 21 st century (1).

However, people age differently, depending on life course exposures to health risks, their lifestyle, genetic makeup, socialeconomic background, and many other factors. To understand how people age requires a nuanced and textured understanding of these determinants. Healthy ageing has received many definitions and interpretations, yet without achieving a consensus. The concept of healthy ageing was a matter of concern since antiquity. Among ancient Greek philosophers, Plato holds out hope for healthy ageing through spirituality; in particular he wrote: "The spiritual eyesight improves as the physical eyesight declines" (2). Healthy ageing is described by the World Health Organization as a process of developing and maintaining the functional ability that enables well-being in older age. Functional ability involves the health-related attributes that make people able to be and to do what they consider valuable. It is made up of the intrinsic individual's ability, the environmental characteristics and the interactions between them while well-being is considered to include domains such as fulfilment, happiness and satisfaction (3). Healthy ageing, in short, is both a matter of how long and how well one lives. Consequently, determinants of health and disease, in addition to risk factors, should be identified to better understand healthy ageing. Discerning patterns of healthy ageing is a substantial challenge that so far has not been systematically attempted in all its complexity. Another challenge is that healthy ageing trajectories are likely to be shaped by study's effects. For example, detected increases in incidence of diabetes may mean that future cohorts will age differently than previous cohorts.

Thus, the present work aimed to explore determinants of healthy ageing among apparently healthy adults; and the associa- 
tion between a healthy ageing score and 10-year cardiovascular disease (CVD) incidence. The research hypothesis, from public health and policy perspective, was that the early detection of a population's level of healthy ageing determinants could guide the efforts of health authorities in early and better prevention of CVD.

\section{MATERIALS AND METHODS}

\section{Sampling Procedure at Baseline Examination}

The "ATTICA" study is a health and nutrition survey that was carried out in the province of Attica during 2001-2002. The invitation clarified that people with history of CVD or living in institutions or having chronic viral infections should not participate in the sampling. Of the 4,056 invited individuals 3,042 agreed to participate ( $75 \%$ participation rate), aged $18-89$ years. However, data for the present analysis were obtained from a sub-sample of 853 participants ( 453 men, aged $45 \pm 13$ years; 400 women, aged $44 \pm 18$ years) that had complete psychological evaluation. No differences regarding age, gender and CVD risk factors profile were observed when the aforementioned sub-group of participants was compared with the entire sample (all p-values $>0.20$ ).

\section{Baseline Measurements}

The baseline evaluation included information about socio-demographic characteristics (age, sex, marital status, mean annual income, and years of education), personal and family history of hypertension, hypercholesterolemia and diabetes, family history of CVD, dietary and other lifestyle habits (i.e., smoking status and physical activity). The evaluation of the nutritional habits was based on a validated semi-quantitative food-frequency questionnaire (4), the EPIC-Greek questionnaire, that was kindly provided by the Unit of Nutrition of Athens Medical School; overall dietary habits were evaluated using the MedDietScore (theoretical range 0-55) that incorporates the inherent characteristics of the Mediterranean diet (5). For the ascertainment of physical activity status, the International Physical Activity Questionnaire (IPAQ) was used (6), as an index of weekly energy expenditure using frequency (times per week), duration (in minutes per occasion) and intensity of sports or other habits related to physical activity (in expended calories per occasion). Participants who did not report any physical activities were defined as physically inactive and the rest as physically active. Basal Metabolic Rate (BMR) was estimated using the Schofield equations for the prediction of BMR (7), adopted by the $2004 \mathrm{FAO} / \mathrm{WHO} / \mathrm{UNU}$ report. Obesity was defined as body mass index (BMI) greater than $29.9 \mathrm{Kg} / \mathrm{m}^{2}$. Waist (in $\mathrm{cm}$ ) and hip (in $\mathrm{cm}$ ) circumferences were also measured and the waist-to-hip ratio (WHR) was calculated (waist-to-hip ratio greater or equal to 0.95 in men and 0.80 in women was considered as abnormal, i.e. a measure of central fat). Current smokers were defined as those who were smoking at least one cigarette per day or had recently stopped smoking (during a year); the rest of the participants were defined as noncurrent smokers. Therefore, participants who were smoking at least one cigarette per day at baseline or in the past, were defined as current or former smokers. The baseline evaluation also included a series of clinical and biochemical measurements.
Arterial blood pressure was measured at the end of the physical examination with subject in sitting position and at least 30 minutes at rest. Participants whose average of three consecutive measurements of blood pressure levels were greater or equal to $140 \mathrm{and} /$ or $90 \mathrm{mmHg}$, or reported that they were under antihypertensive medication, were classified as having hypertension. Blood samples were collected from the antecubital vein early in the morning after 12 hours of fasting and alcohol abstinence. Hypercholesterolemia was defined as total cholesterol levels greater than $220 \mathrm{mg} / \mathrm{dl}$ or the use of lipid lowering medication and diabetes mellitus as a fasting blood sugar $>125 \mathrm{mg} / \mathrm{dl}$ or the use of antidiabetic medication. High sensitivity C-reactive protein (CRP) was assayed by particle-enhanced immunonephelometry (N Latex, Dade-Behring Marburg GmbH, Marburg, Germany). The Greek translation of the 20-item self-report State-Trait Anxiety Inventory (STAI), state version was used to evaluate levels of anxiety (8) while depressive symptomatology was assessed using a translated and validated version of the Zung Self-Rating Depression Scale (ZDRS) (9).

For the purposes of the present work analysis, an index that incorporated healthy ageing factors, the Healthy Ageing Index (HAI), was developed based on the concept that has been already proposed and validated by previous investigators (10). HAI was calculated based on socioeconomic (years of education and financial status), bio-clinical (presence of hypertension, hypercholesterolemia, diabetes, obesity, and BMI classification), psychological (depression status) and lifestyle characteristics (MedDietScore and physical activity). HAI's theoretical range was $0-7$. Higher scores correspond to higher levels of healthy ageing.

As a proxy of risk for fatal CVD events, the HellenicSCORE was calculated based on the baseline information retrieved from the participants. HellenicSCORE is a recalibration of the European Society of Cardiology (ESC) SCORE (11) that measures absolute overall risk of an individual to develop a fatal CVD event during the next 10-year of his/her life (values between $5-10 \%$ are considered as moderate risk and values $>10 \%$ are considered as high risk) (12).

\section{Follow-up Examination}

During 2011-12, the 10-year follow-up was performed. In order to participate in the follow-up all participants were initially appointed through telephone calls ( $89 \%$ of the participants) or face-to-face interviews when their telephone number was not available. Of the initially studied participants at baseline examination, $85 \%$ were found during the follow-up. No differences were reported regarding the distribution of $\operatorname{sex}(p=0.613)$, as well as anxiety $(p=0.083)$ and depression levels $(p=0.173)$ between the participants that were found to follow-up and the participants that were lost to follow-up.

Fatal or non-fatal CVD events during the 10 -year follow up were defined as follows: development of coronary heart disease (CHD) (i.e., myocardial infarction, angina pectoris, other identified forms of ischaemia WHO-ICD coding 410-414.9, 427.2, 427.6, heart failure of different types, and chronic arrhythmias WHO-ICD coding 400.0-404.9, 427.0-427.5, 427.9), or development of stroke (WHO-ICD coding 430-438). Regarding CVD ascertainment medical records and hospital data were obtained. The adjudication was made by trained physicians and followed 
the International Statistical Classification of Diseases and Related Health Problems 10th Revision.

\section{Research Ethics}

The study has been approved by the Institutional Ethics Committee. All participants have been informed about the aims and procedures and agreed to participate providing written consent.

Further details about the aims and procedures of the ATTICA study may be found in the methodology paper (13).

\section{Statistical Analysis}

Unadjusted associations between various demographic, anthropometric, as well as clinical characteristics and 10-year CVD incidence were performed using Student's t-test (for the continuous and normally distributed variables), Mann-Whitney $\mathrm{U}$ test (for the continuous variables with skewed distribution) and Pearson chi-square test (for the categorical variables). Normality assumption was assessed via P-P plots. Continuous and normally distributed characteristics are presented as mean values \pm standard deviation or as median values (minimummaximum) in case of skewed distribution. Categorical variables are presented as frequencies. To evaluate the association between various socio-demographic, bio-clinical and nutritional factors (independent variables) and the level of healthy ageing (dependent outcome) nested multiple linear regression models were estimated. Colinearity was tested using the variance inflation factor criterion, while the assumption of homoscedasticity was tested by plotting the scatter plot of standardized residuals over the predicted score values. Results from linear regression models are presented as b-coefficients and their $95 \%$ confidence intervals. Nested multiple logistic regression models were developed in order to evaluate the association between the 10 -year CVD risk (dependent outcome) and the level of healthy ageing at baseline (exposure variable), after adjusting for various participant characteristics (age, sex, anxiety levels, smoking habits, sedentariness, alcohol consumption, and family history of CVD). The Hosmer-Lemeshow statistic was applied for the assessment of model's goodness-of-fit. Furthermore, in order to evaluate the additive effect of HAI on the HellenicSCORE predictive ability for 10-year CVD risk, two logistic regression models were applied, with the 10-year CVD risk as the dependent outcome. In the first one, only HellenicSCORE was introduced as predictor, while in the second one, HAI was additionally comprised (data not shown). Then, the corresponding ROC curves were constructed, and the $\mathrm{C}$-statistics were calculated (C-statistic is a measure of accuracy of each estimated model). All reported p-values were based on two-sided tests. SPSS 20 software (SPSS Inc., Chicago, IL, USA) was used for all statistical calculations.

Table 1. Participants' demographic, anthropometric and clinical characteristics by 10-year cardiovascular disease incidence $(N=845)$

\begin{tabular}{|c|c|c|c|}
\hline \multirow{2}{*}{ Baseline information } & \multicolumn{3}{|c|}{ Cardiovascular Disease (10-year Follow-up) } \\
\hline & No $(n=802)$ & Yes $(n=43)$ & $\mathrm{p}$ \\
\hline Healthy Aging Index (range 0-7) & $4.0 \pm 0.77$ & $3.5 \pm 0.93$ & 0.005 \\
\hline Gender (male) & $49 \%$ & $72 \%$ & 0.004 \\
\hline Age (years) & $38 \pm 11$ & $48 \pm 13$ & $<0.001$ \\
\hline Marital status (married) & $62 \%$ & $65 \%$ & 0.637 \\
\hline Current or former smoker (yes) & $57 \%$ & $67 \%$ & 0.172 \\
\hline Physical activity (yes) & $38 \%$ & $40 \%$ & 0.798 \\
\hline \multicolumn{3}{|l|}{ Alcohol consumption } & 0.217 \\
\hline$<4 \mathrm{~g}$ of ethanol/day & $50 \%$ & $60 \%$ & \\
\hline $4-25 \mathrm{~g}$ of ethanol/day & $43 \%$ & $29 \%$ & \\
\hline$>25 \mathrm{~g}$ of ethanol/day & $7 \%$ & $11 \%$ & \\
\hline Coffee consumption (ml/day) & $50(0,300)$ & $150(0,250)$ & 0.276 \\
\hline Spielberger State Anxiety Inventory (range 20-80) & $41 \pm 12$ & $44 \pm 13$ & 0.086 \\
\hline Abnormal waist to hip ratio & $45 \%$ & $61 \%$ & 0.053 \\
\hline Triglycerides (mg/dl) & $83(27,587)$ & $119(48,568)$ & $<0.001$ \\
\hline C-reactive protein (mg/L) & $0.95(0.01,13.1)$ & $1.5(0.02,14.8)$ & 0.132 \\
\hline Basic metabolic rhythm (kcal/day) & $1575(1102,2553)$ & $1693(1232,2314)$ & 0.049 \\
\hline History of hypertension (yes) & $54 \%$ & $61 \%$ & 0.537 \\
\hline History of diabetes mellitus (yes) & $29 \%$ & $39 \%$ & 0.308 \\
\hline History of hypercholesterolemia (yes) & $40 \%$ & $57 \%$ & 0.132 \\
\hline Family history of CVD (yes) & $39 \%$ & $75 \%$ & 0.001 \\
\hline HellenicSCORE (absolute 10-year CVD risk) & $31(1,25)$ & $2.4(0,7.9)$ & $<0.001$ \\
\hline
\end{tabular}

Results are presented as relative frequencies (\%), mean \pm SD, median (min, max) 


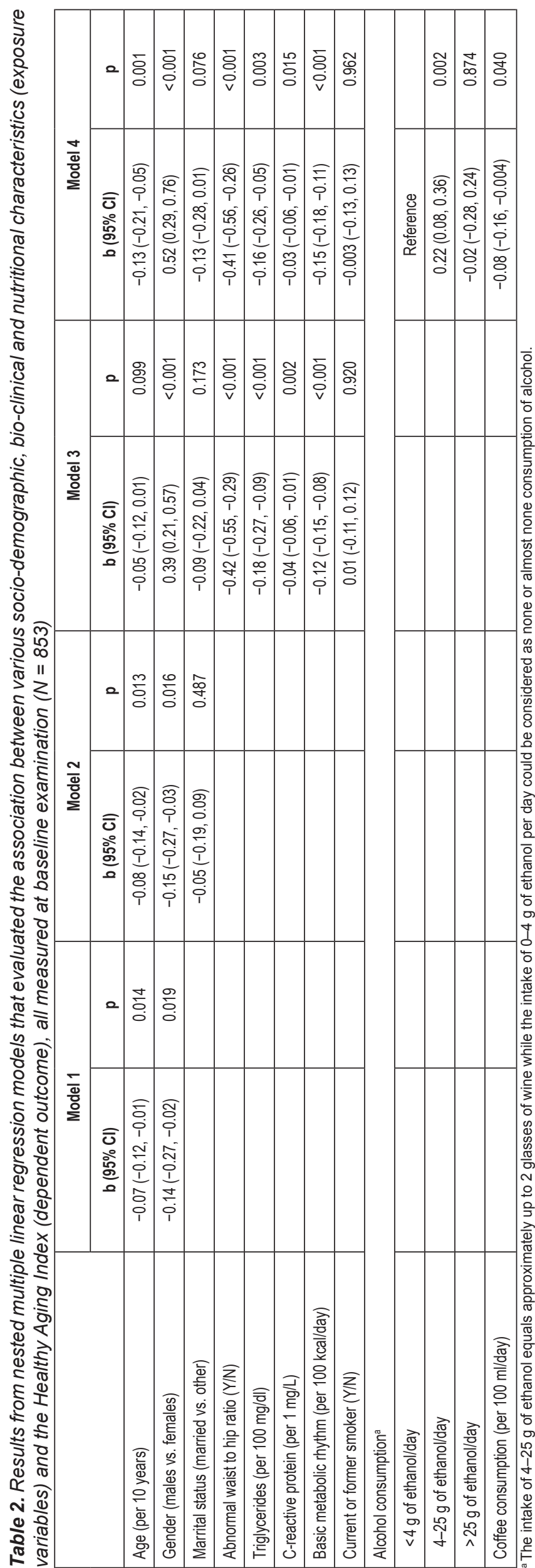

\section{RESULTS}

During the 10-year (2002-2012) follow-up period, the fatal or non-fatal CVD incidence was 43 cases (5.1\%); of them 31 cases $(72 \%)$ were men and 12 cases $(28 \%)$ were women (p for gender difference 0.004$)$. Of the total CVD events, only 2 (5\%) were fatal. In the unadjusted analysis, HAI was inversely associated with CVD $(p=0.005)$. Higher CVD incidence rates were observed among males $(p=0.004)$, older individuals $(p<0.001)$, and participants with central obesity (0.053), family history of CVD $(p=0.001)$, and higher levels of triglycerides $(p<0.001)$ or higher BMR (0.049) (Table 1).

\section{Determinants of Healthy Ageing}

Regarding the age-gender adjusted determinants of healthy ageing, abnormal waist to hip ratio $(\mathrm{p}<0.001)$ and reduced BMR $(\mathrm{p}<0.001)$ were inversely associated with HAI score. Negative association was also found regarding the levels of triglycerides $(p=0.003)$ and CRP $(p=0.02)$ (Table 2). Furthermore, coffee consumption was inversely associated with HAI $(\mathrm{p}=0.04)$, while a positive association was observed with moderate alcohol consumption compared to none or almost none $(\mathrm{p}=0.002)$ and male gender $(\mathrm{p}<0.001)$.

\section{Healthy Ageing and 10-year CVD Risk}

The Healthy Ageing Index was found to be a strong, protective factor against 10 -year CVD incidence $(p=0.01)$ (Table 3$)$. This association remained significant $(p=0.01)$ even after adjusting for other psychological (anxiety levels) and lifestyle characteristics (smoking habits, physical activity status and alcohol consumption) of the participants. Specifically, 14\% (1/7 units) increase in HAI was associated with 53\% reduction in 10-year CVD risk, after adjusting for gender, age, anxiety level, smoking habits, physical activity, and alcohol consumption. All of the aforementioned covariates are established risk factors for CVD development (14-16).

Furthermore, the predictive ability of HAI as compared to HellenicSCORE was also tested by comparing AUC of the ROC curve in the age-sex adjusted model that included only HAI versus AUC in the model that included only HellenicSCORE. HAI demonstrated an additive effect of $0.8 \%$ on the HellenicSCORE predictive ability for 10-year CVD risk.

\section{DISCUSSION}

In the present work healthy ageing was strongly associated with the CVD risk of apparently healthy individuals at baseline examination. Apparently, healthy ageing was strongly and independently of other well-known lifestyle and biological risk factors associated with CVD development. In addition, healthy ageing contributed to the better identification of future CVD events since it increased the predictive ability of the HellenicSCORE in the model that evaluated 10 -year CVD risk. This finding implies HAI's potential adequacy of being used as a reliable and valid alternative in the early detection of the population at risk. The precise tracking of high-risk groups is a matter of major concern as the benefits of prevention versus treatment are beyond doubt. 
Table 3. Results from nested logistic regression models that evaluated the association between the Healthy Aging Index (main effect, exposure variable) and the 10-year risk of CVD (dependent outcome), after adjusting for various socio-demographic, bio-clinical and nutritional characteristics measured at baseline $(N=853)$

\begin{tabular}{|c|c|c|c|c|c|c|}
\hline & \multicolumn{2}{|c|}{ Model 1} & \multicolumn{2}{|c|}{ Model 2} & \multicolumn{2}{|c|}{ Model 3} \\
\hline & b $(95 \% \mathrm{Cl})$ & $\mathrm{p}$ & b $(95 \% \mathrm{Cl})$ & $\mathrm{p}$ & b $(95 \% \mathrm{Cl})$ & $\mathrm{p}$ \\
\hline Healthy Aging Index a (per 1 unit) & $0.55(0.36,0.85)$ & 0.006 & $0.47(0.28,0.80)$ & 0.005 & $0.77(0.36,1.66)$ & 0.502 \\
\hline Gender (males vs. females) & $2.08(1.00,4.31)$ & 0.050 & $3.00(1.22,7.36)$ & 0.016 & $3.35(0.90,12.55)$ & 0.072 \\
\hline Age (per 10 years) & $2.20(1.54,3.16)$ & $<0.001$ & $1.95(1.30,2.95)$ & 0.001 & $1.50(0.78,2.88)$ & 0.224 \\
\hline STAI (per 1 unit) ${ }^{b}$ & & & $1.01(0.98,1.05)$ & 0.519 & $1.06(1.01,1.11)$ & 0.030 \\
\hline Current or former smoker (Y/N) & & & $1.39(0.61,3.21)$ & 0.435 & $2.81(0.78,10.12)$ & 0.113 \\
\hline Sitting minutes/day (per 60 min) & & & $0.84(0.70,1.01)$ & 0.066 & $0.85(0.65,1.11)$ & 0.228 \\
\hline Alcohol consumption ${ }^{c}$ & & & & 0.092 & & 0.023 \\
\hline$<4 \mathrm{~g}$ of ethanol/day & & & Reference & & Reference & \\
\hline $4-25 \mathrm{~g}$ of ethanol/day & & & $0.37(0.15,0.91)$ & 0.029 & $0.11(0.02,0.54)$ & 0.006 \\
\hline$>25 \mathrm{~g}$ of ethanol/day & & & $0.58(0.15,2.31)$ & 0.440 & $0.50(0.09,2.77)$ & 0.425 \\
\hline Family history of CVD (Y/N) & & & & & $4.59(1.31,16)$ & 0.017 \\
\hline
\end{tabular}

a Healthy Ageing Index is calculated based on socio-economical, bio-clinical (presence of hypertension, hypercholesterolemia, diabetes and obesity and BMI classification), psychological (i.e., depression status) and lifestyle characteristics (MedDietScore and physical activity). ${ }^{\mathrm{b}}$ Spielberger State Anxiety Inventory. ${ }^{\mathrm{c}}$ The intake of $4-25 \mathrm{~g}$ of ethanol equals approximately up to 2 glasses of wine while the intake of $0-4 \mathrm{~g}$ of ethanol per day could be considered as none or almost none consumption of alcohol.

In addition, healthy ageing determinants were also studied here. Low triglycerides and CRP levels, reduced BMR, normal waist to hip ratio, moderate alcohol drinking (up to 2 glasses daily) as compared to the substantial abstention from alcohol, were associated with healthy aging. Previous research findings have already underlined the association between healthy aging and moderate alcohol drinking (17), as well as BMR and health status at a later stage of life. Specifically, BMR has been proposed as a significant risk factor for mortality, independent of secular trends and other well-recognized risk factors, such as age, body mass index, smoking, white blood cell count, and diabetes in a research of a 40 -year follow-up period (18). Moreover, triglycerides and CRP levels have not only been identified as risk factors for CVD $(19,20)$ or other chronic diseases, but may also be indicative for impaired cognitive performance $(21,22)$, related not only to shorter life expectancy, but also to inferior cognition in longevity. Regular exercise and higher adherence to Mediterranean diet, i.e. two of the HAI structural components, have both been associated with lower CRP or/and triglycerides levels $(5,23)$. Equally remarkable is the fact that CRP levels have been also associated with depressed mood (24), which is another structural factor of ageing.

In contrast, increased coffee drinking was associated with reduced healthy ageing score. According to recent literature, coffee drinking increases the experience of stress by stimulating the release of stress hormones. The continuous presence of these hormones not only has a damaging effect on a number of different physiological systems, but can also accelerate the ageing process. When stress hormones are chronically elevated, as a result of our intense lifestyle as well as a consequence of ingesting coffee and caffeine, the body's normal repair mechanisms are inhibited, thereby accelerating the ageing process (25). This could be a possible mechanism explaining the identified inverse association between coffee drinking and healthy ageing.

Alongside, central obesity, as it emerged through the evaluation of the waist to hip ratio in the present work, is another parameter that has been linked to various health outcomes while it has been also suggested that a larger WHR may be related to neurodegenerative, vascular, or metabolic processes that affect brain structures underlying cognitive decline and dementia (26). The identified negative association between abdominal fat and healthy ageing could be partially attributed to the fact that in healthy ageing calculation somatometric characteristics are incorporated, but visceral fat is also known to be associated with dietary patterns (5), financial hardship and low educational level (27).

Nevertheless, it remains unquestionable that people age differently, depending on life course exposures to health risks, different socioeconomic backgrounds, and many other factors. There are, in short, different ageing pathways or trajectories. To understand healthy ageing, therefore, requires a nuanced and textured understanding of these pathways, as well as the determinants of healthy ageing. Specifically, we need to know if there are recognizable patterns of healthy ageing trajectories across the population. Identifying these patterns opens the door to focused interventions, both at the population and clinical levels, which optimize healthy ageing.

As mortality statistics suggest that CVD remains the leading cause of death among Europeans and around the world, a large number of epidemiological studies aim to identify predictors of CVD. In this context, the main finding of the present work, i.e. the strong association between healthy ageing and 10-year CVD risk is an observation that deserves further attention for public health (28). HAI could be possibly used as an additive or even as an alternative approach of overall CVD risk estimation, in order to early detect the population at risk.

\section{Limitations}

Despite the aforementioned, it should not be overlooked that psychological assessment was addressed to a rather small part of the entire ATTICA Study sample, a fact that probably constitutes a limitation of the present work. Considering that in HAI calculation, psychological assessment (i.e. depression) is incorporated, 
the sample size was unavoidably restricted, limiting perhaps the possibility of clearer picture.

\section{CONCLUSION}

The fact that healthy ageing may constitute a single and simple factor that may predict future CVD events, provides a new direction for the prevention strategies at population level. Because of the alarming rates of CVD globally, identifying patterns and determinants of healthy ageing opens the door to focused public health and personalized interventions to optimize healthy ageing, both at the population and individual levels.

\section{Acknowledgements}

The authors would like to thank the ATTICA study group of investigators: Yannis Skoumas, Natassa Katinioti, Labros Papadimitriou, Constantina Masoura, Spiros Vellas, Yannis Lentzas, Manolis Kambaxis, Konstadina Palliou, Vassiliki Metaxa, Agathi Ntzouvani, Dimitris Mpougatsas, Nikolaos Skourlis, Christina Papanikolaou, Georgia-Maria Kouli, Aimilia Christou, Adella Zana, Maria Ntertimani, Aikaterini Kalogeropoulou, Evangelia Pitaraki, Alexandros Laskaris, Mihail Hatzigeorgiou and Athanasios Grekas for their assistance in the initial physical examination and follow-up evaluation, Efi Tsetsekou for her assistance in psychological evaluation, as well as the laboratory team: Carmen Vassiliadou and George Dedoussis (genetic analysis), Marina Toutouza-Giotsa, Constadina Tselika and Sia Poulopoulou (biochemical analysis) and Maria Toutouza for the database management.

\section{Conflict of Interest}

None declared

\section{Funding}

Demosthenes Panagiotakos and Ekavi Georgousopoulou received research grants by the Coca-Cola Company. The ATTICA study is supported by research grants from the Hellenic Cardiology Society (HCS2002) and the Hellenic Atherosclerosis Society (HAS2003). DBP and NK are funded within the Aging Trajectories of Health: Longitudinal Opportunities and Synergies (ATHLOS) project (European Union's Horizon 2020 research and innovation program) under grant agreement No 635316.

\section{REFERENCES}

1. European Commission. The demographic future of Europe - from challenge to opportunity. Luxembourg: Office for Official Publications of the European Communities; 2006.

2. Zubko A. Treasury of spiritual wisdom. San Diego: Blue Dove Press; 1998.

3. World Health Organization. World report on ageing and health. Geneva: WHO; 2015.

4. Katsouyanni K, Rimm EB, Gnardellis C, Trichopoulos D, Polychronopoulos E, Trichopoulou A. Reproducibility and relative validity of an extensive semi-quantitative food frequency questionnaire using dietary records and biochemical markers among Greek schoolteachers. Int J Epidemiol. 1997;26 Suppl 1:118-27.

5. Panagiotakos DB, Pitsavos C, Stefanadis C. Dietary patterns: a Mediterranean diet score and its relation to clinical and biological markers of cardiovascular disease risk. Nutr Metab Cardiovasc Dis. 2006;16(8):55968.

6. Papathanasiou G, Georgoudis G, Papandreou M, Spyropoulos P, Georgakopoulos D, Kalfakakou V, et al. Reliability measures of the short International Physical Activity Questionnaire (IPAQ) in Greek young adults. Hellenic J Cardiol. 2009;50(4):283-94.
7. Schofield WN. Predicting basal metabolic rate, new standards and review of previous work. Hum Nutr Clin Nutr. 1985;39 Suppl 1:5-41.

8. Fountoulakis KN, Papadopoulou M, Kleanthous S, Papadoupoulou A, Bizeli V, Nimatoudis I, et al. Reliability and psychometric properties of the Greek translation of the Sate-Trate Anxiety Inventory form Y: preliminary data. Ann Gen Psychiatry. 2006 Jan 31;5:2. doi: 10.1186/1744-859X-5-2.

9. Fountoulakis KN, Iacovides A, Samolis S, Kleanthous S, Kaprinis SG, Kaprinis GS, et al. Reliability, validity and psychometric properties of the Greek translation of the zung depression rating scale. BMC Psychiatry. 2001;1:6. doi: 10.1186/1471-244X-1-6.

10. Tyrovolas S, Haro JM, Mariolis A, Piscopo S, Valacchi G, Tsakountakis $\mathrm{N}$, et al. Successful aging, dietary habits and health status of elderly individuals: a k-dimensional approach within the multi-national MEDIS study. Exp Gerontol. 2014;60:57-63.

11. Panagiotakos DB, Fitzgerald AP, Pitsavos C, Pipilis A, Graham I, Stefanadis C. Statistical modelling of 10-year fatal cardiovascular disease risk in Greece: the HellenicSCORE (a calibration of the ESC SCORE project). Hellenic J Cardiol. 2007;48(2):55-63.

12. Conroy RM, Pyörälä K, Fitzgerald AP, Sans S, Menotti A, De Backer $\mathrm{G}$, et al.; SCORE project group. Estimation of ten-year risk of fatal cardiovascular disease in Europe: the SCORE project. Eur Heart J. 2003;24(11):987-1003.

13. Pitsavos C, Panagiotakos DB, Chrysohoou C, Stefanadis C. Epidemiology of cardiovascular risk factors in Greece: aims, design and baseline characteristics of the ATTICA study. BMC Public Health. 2003;3:32. doi: 10.1186/1471-2458-3-32.

14. Pitsavos C, Panagiotakos DB, Papageorgiou C, Tsetsekou E, Soldatos C, Stefanadis C. Anxiety in relation to inflammation and coagulation markers, among healthy adults: the ATTICA study. Atherosclerosis. 2006;185(2):320-6.

15. Kamphuis CB, Turrell G, Giskes K, Mackenbach JP, van Lenthe FJ. Socioeconomic inequalities in cardiovascular mortality and the role of childhood socioeconomic conditions and adulthood risk factors: a prospective cohort study with 17-years of follow up. BMC Public Health. 2012 Dec 5;12:1045. doi: 10.1186/1471-2458-12-1045.

16. Panagiotakos DB, Georgousopoulou EN, Pitsavos C, Chrysohoou C, Metaxa V, Georgiopoulos GA, et al.; ATTICA Study group. Ten-year (2002-2012) cardiovascular disease incidence and all-cause mortality, in urban Greek population: the ATTICA Study. Int J Cardiol. 2015;180:17884.

17. Genchev GD, Georgieva LM, Weijenberg MP, Powles JW. Does alcohol protect against ischaemic heart disease in Bulgaria? A case-control study of non-fatal myocardial infarction in Sofia. Cent Eur J Public Health. 2001;9(2):83-6.

18. Ruggiero C, Metter EJ, Melenovsky V, Cherubini A, Najjar SS, Ble A, et al. High basal metabolic rate is a risk factor for mortality: the Baltimore Longitudinal Study of Aging. J Gerontol A Biol Sci Med Sci. 2008;63(7):698-706.

19. Đokić B, Đonović N, Tadić B, Nikolić D. Factors and estimation of risk for cardiovascular diseases among patients in primary health care in Central Serbia. Cent Eur J Public Health. 2015;23(3):195-9.

20. Lagrand WK, Visser CA, Hermens WT, Niessen HW, Verheugt FW, Wolbink GJ, et al. C-reactive protein as a cardiovascular risk factor: more than an epiphenomenon? Circulation. 1999;100(1):96-102.

21. Atzmon G, Gabriely I, Greiner W, Davidson D, Schechter C, Barzilai N. Plasma HDL levels highly correlate with cognitive function in exceptional longevity. J Gerontol A Biol Sci Med Sci. 2002;57(11):M712-5.

22. Teunissen CE, van Boxtel MP, Bosma H, Bosmans E, Delanghe J, De Bruijn $\mathrm{C}$, et al. Inflammation markers in relation to cognition in a healthy aging population. J Neuroimmunol. 2003;134(1-2):142-50.

23. Lira FS, Rosa Neto JC, Antunes BM, Fernandes RA. The relationship between inflammation, dyslipidemia and physical exercise: from the epidemiological to molecular approach. Curr Diabetes Rev. 2014;10(6):391-6.

24. Penninx BW, Kritchevsky SB, Yaffe K, Newman AB, Simonsick EM, Rubin $\mathrm{S}$, et al. Inflammatory markers and depressed mood in older persons: results from the Health, Aging and Body Composition study. Biol Psychiatry. 2003;54(5):566-72.

25. Raber J. Detrimental effects of chronic hypothalamic-pituitary-adrenal axis activation. From obesity to memory deficits. Mol Neurobiol. 1998;18(1):1-22.

26. Jagust W, Harvey D, Mungas D, Haan M. Central obesity and the aging brain. Arch Neurol. 2005;62(10):1545-8.

27. Pikhart H, Bobak M, Malyutina S, Pajak A, Kubínová R, Marmot M. Obesity and education in three countries of the Central and Eastern Europe: the HAPIEE study. Cent Eur J Public Health. 2007;15(4):140-2. 
28. Gorenoi V, Hagen A. Overview of risk-estimation tools for primary prevention of cardiovascular diseases in European populations. Cent Eur J Public Health. 2015;23(2):91-9. 